\title{
Identifying Potential High-Risk Medication Errors Using Telepharmacy and a Web-Based Survey Tool
}

Nishat Afreen Huq, BS ${ }^{1}$; Eimeira Padilla-Tolentino, PharmD, PhD²; Brandy McGinnis, PharmD, BCPS ${ }^{3}$

${ }^{1}$ Pharmacy Intern, PharmD Candidate 2021, University of Texas at Austin College of Pharmacy, and Pharmacy Technician, Ascension

Seton Department of Pharmacy

${ }^{2}$ Ascension Texas Department of Research, and Clinical Instructor, University of Texas at Austin College of Pharmacy

${ }^{3}$ Area Director of Continuity of Care, Ascension Texas Department of Pharmacy, and Clinical Instructor, University of Texas at Austin College of Pharmacy

\section{Abstract}

Background and Introduction: Obtaining patient medication histories during emergency department (ED) admissions is an important step towards identifying potential errors that could otherwise remain in the patient's active medication list. This is a descriptive report of a standardized, electronic data collection tool created to document potential medication errors in patients receiving high-risk medications during ED admissions.

Materials and Methods: Trained pharmacy technicians completed a survey following medication history collection using a secure web platform called REDCap ${ }^{\circledR}$. Data collected included patient-specific information, the number and type of high-risk medications, and potential medication errors identified in the collection process.

Results: During a pilot period of April 2019 to October 2020, 191 patient records were completed using the survey tool. Out of a total of 1088 medications recorded, 41\% were considered high-risk medications. $42 \%$ of potential medication errors were classified as highrisk medication errors. Results from this survey tool demonstrated that 58\% of high-risk medication orders could potentially result in a medication error that can be carried through patient admission and discharge.

Discussion: Accurate medication history and transitions of care can significantly impact patient quality of life. The cost of addressing a medication related-adverse event is also substantial. Based on published reports, annual gross savings to a hospital is estimated to be $\$ 4532$ per harmful error in 2020, after adjusting for inflation. This equated to approximately $\$ 1,182,852$ in estimated savings for Ascension Texas in 18 months. Nationwide, preventing potential medication errors in an outpatient setting can save on average \$3.5 billion per year.

Conclusion: This web-based survey tool has improved the quality and efficiency of potential error identification during medication history collection by pharmacy technicians. This information can be easily retrieved and aid in discussions regarding medication reconciliation at the leadership level and impact patient treatment outcomes by developing virtual processes that may result in fewer medication related events.

Keywords: Pharmacy, Telepharmacy, Telehealth, Web-based, Electronic Health Records, Medication errors, High-risk medications, High-risk medication errors

\section{Introduction}

Medication-related adverse events and errors are responsible for a significant portion of patient morbidity, mortality, and hospital admissions, all resulting in increased healthcare expenditures and decreased patient quality of life. ${ }^{1-5}$ Adverse drug events (ADEs) result in over 700,000 injuries or deaths in U.S. hospitals per year, and up to half are associated with preventable medication errors, each of which cost hospitals between $\$ 2852$ and $\$ 8116$ in extra expenses. ${ }^{1-3}$ Evidence supports that over $27 \%$ of hospital prescribing errors and at least $50 \%$ of medication discrepancies during discharge are associated with admission discrepancies and incorrect medication histories. ${ }^{4}$ Further increasing the chance of medication-related errors is polypharmacy, which is the use of

Corresponding author: Brandy McGinnis, PharmD, BCPS Area Director of Continuity of Care (TX Region) GoodHealth Solution Center 7701 Metropolis Dr. BLDG 13, Suite \#100 Austin, TX 78744; Office: 512-103-5223 (ext. within Seton 35223) Cell: 512-745-2291; Email: bmcginnis@ascension.org multiple medications to treat a single patient, a phenomenon that has been increasing in the United States for several years. ${ }^{5-7}$ Older adults aged $\geq 65$ years of age tend to have a higher number of chronic conditions and therefore increased use of medications. ${ }^{5-7}$ Polypharmacy is associated with a higher risk of ADEs, with significant impact on mortality and likelihood of hospitalization. ${ }^{5-7}$ Preventing such potential medication errors as a result of polypharmacy or medication discrepancies in an outpatient setting can save an average of $\$ 3.5$ billion per year nationwide. ${ }^{1,3,8}$ Therefore obtaining accurate medication histories is especially important in the admission of patients in the emergency department (ED), and documentation of interventions taken are crucial in communicating with health-care providers to raise awareness of patients with more complicated medication regimens. This process can resolve errors during transitions of care, identify medication-related adverse events, and medication nonadherence. ${ }^{3,5}$ In particular, medication errors that cause significant harm, prolonged hospitalizations, or even death are associated with high-risk (HR) medications. ${ }^{5}$ Therefore identifying and resolving potential high-risk medication errors before a patient is sent to a hospital unit for further care helps 
ensure those errors are not being carried through discharge, during which up to $75 \%$ of potential adverse drug events occur. ${ }^{5,9}$

Pharmacists have unique training in therapeutics and the ability to perform comprehensive medication therapy management. Although current literature demonstrates the important role pharmacists play in medication reconciliation, resource limitations often occur and hinder expansion of these services. ${ }^{5,10-15}$ Utilization of pharmacy technicians to perform medication histories has quickly become a unique and innovative way to provide high-quality care with reduced resources, and has proven to be faster and more accurate than relying on non-pharmacy personnel such as nurses or medical assistants. Studies have demonstrated significant reductions in medication errors and drug interactions when instituting a program utilizing pharmacy technicians to obtain medication histories with pharmacist oversight. ${ }^{5,11-12}$

A centralized call center model was established in 2015 in the Ascension Texas network of hospitals in Central Texas, enabling pharmacy technicians to work remotely using virtual technology video-conferencing via mobile carts to collect medication histories from patients in EDs. This program was initiated to address staffing shortages, a decline in medication history completion rates, and medication errors identified during medication history collection. Implementation of this technology has allowed technicians to complete medication histories from a remote location and have access to patients at six Ascension Texas hospitals by using a bed board system that updates the arrival and admission of new patients in the ED. Technicians communicate with nurses and ED staff via phone to coordinate the delivery of the mobile-cart to the patient's bedside. By interviewing the patient through video conferencing, the technicians could see patients' medication bottles, home medication lists, and communicate with the patient directly. The technicians accessed the patient's electronic medical record (EMR) to update the home medication list, and reviewed an external prescription database to view past prescription refills at retail pharmacies. They could additionally contact provider offices, outside clinics, and community pharmacies as appropriate. Services are currently provided by six full-time certified pharmacy technicians who are trained in collecting medication histories. The hospitals included in this service are Dell Seton Medical Center (DSMC), Ascension Seton Medical Center Austin (ASMCA), Ascension Seton Williamson (ASW), Ascension Seton Hays (ASH), Ascension Seton Northwest (ASNW) and Ascension Providence Health Center Hospital. ${ }^{5}$

In addition to their normal workflow the pharmacy technicians were instructed to track and identify medication errors found in the collection process, which were categorized into five types of potential errors: extra (patient was not currently taking a medication listed in the medication list), omitted (patient was currently taking a medication that was not documented in the medication list), duplicate (two plus entries of the same medication or medication class), incomplete (medication listed was missing the name, dose, or frequency of use), and incorrect (medication listed had a different name, dose, or frequency of use) errors. ${ }^{5}$ Identification of such errors during the medication history process was documented by each technician manually on paper, and retrospectively analyzed by an overseeing pharmacist. Each error was classified as a high-risk (HR) or nonhigh-risk (non-HR) medication error. Due to resource limitations, the call center was unable to collect this information in a manner that could be reported on a regular basis without increasing staffing demands or resources. ${ }^{5}$

The goal of this pilot study was to develop an innovative survey tool that standardized the process of recording types of HR medications and medication errors found during medication history collection. Additionally, the tool would allow documentation during workflow without requiring further time and resources to analyze data at a later time point. This would enable reporting on a monthly basis to the Ascension Texas network of hospitals, from which information could be used to optimize patient care and minimize costly errors that often arise during transitions of care. Successful incorporation of this survey tool into technician workflow could provide a model for healthcare institutions to prioritize the identification and nature of medication errors and impact patient treatment outcomes by developing processes that may result in fewer medication-related adverse events.

\section{Materials and Methods}

A high-risk medication list was developed following a comprehensive literature review using Pubmed. ${ }^{16}$ Key terms used in the search included high-risk medications, high-risk medication errors, and medication-related adverse drug events. Inclusion criteria were systemic medications used in community or outpatient clinic settings. Exclusion criteria were non-systemic medications (i.e. topical or inhaled medications), high-risk medications for populations only with certain medical conditions (ex. statins being contraindicated in pregnancy) and medications used solely in inpatient settings. The exclusion criteria were derived due to the nature of the call-center workflow, during which technicians were only responsible for gathering the patient's current medications used on an outpatient basis, without reporting patients' medical conditions or drug-drug interactions. Lastly, combination drugs were counted as one drug to avoid inflation of total medication numbers. Identified high-risk medications were grouped into categories by drug class or biological system. Broad categories of drugs included were: cardiovascular (specifically renin-angiotensin-aldosterone-system drugs), antiarrhythmic, anticoagulant, non-steroidal anti-inflammatory drugs (NSAIDs), central nervous system (CNS) medications, cytostatic, hypoglycemic, corticosteroid, antirheumatic, immunosuppressant, antibiotic, antiretroviral, antiplatelet, and narrow therapeutic index (NTI) medications, as defined by the 
United States Pharmacopeia (USP) and American Hospital Formulary System (AHSF). ${ }^{17-37}$

Recent studies highlighted that over a third of seniors aged 65 years and older are being prescribed potentially inappropriate medications (PIM) that are associated with adverse events that lead to increased healthcare utilization and treatment costs. 38,39 These included both acute and chronic medications, and were associated with a $21 \%$ increased risk of experiencing an adverse drug event, having to visit the emergency department, or being rehospitalized. ${ }^{38,39}$ Therefore an additional group of medications were added to the HR medication list for those patients over the age of 65, largely incorporated from the 2019 BEERS Criteria, STOPP criteria, Choosing Wisely statements, and Kim et al. ${ }^{40-43}$ These potentially inappropriate medications in the elderly included endocrine, anticholinergic, proton-pump inhibitors (PPIs), electrolyte disturbing, and increased fall/fracture risk medications. ${ }^{40-43}$

Study data were collected by developing a web-based survey tool using Research Electronic Data Capture (REDCap) tools at Ascension Texas. ${ }^{44,45}$ REDCap is a secure, web-based software platform designed to support data collection for research studies. This allowed the standardization of electronic data collection by pharmacy technicians to capture information on the medication categories and error types identified during medication history collection. This web-based tool was created in the form of a branched-logic survey that contained question boxes that populated based upon the technician responses, and termed the Ascension Texas Medication History Survey tool.

The Ascension Texas Medication History Survey tool was designed to be completed in less than five minutes during or after obtaining a medication history via the remote call center system. The survey collected the following data (Figure 1): the patient medical record number (MRN), hospital site (DSMC, ASMCA, ASH, ASNW, ASW, or Providence), date of birth, date of admission, and sources from which the pharmacy technician obtained the necessary information (patient, caregiver, nurse, etc.) in case further clarification was required. Secondly, the technician completed the HR medication section, during which they selected each HR medication encountered during the medication history process by marking checkboxes on a list of over 400 high-risk medications. Technicians could use the "Ctrl $+F^{\prime \prime}$ function on their keyboards to easily search for medication names after a patient's medication history collection. For each selected medication, subsequent branching logic would populate to inquire if there was a medication error associated with that medication, and if so, what type of error (omitted, extra, duplicate, missing, incorrect, or other entry). For example, if a technician selected "oxycodone" as a medication found during a patient's medication history collection, the survey would populate further question boxes based upon the technician's responses. The first question box would ask if there was a medication error associated with oxycodone. If selected "no", no further questions would appear. If "yes", a subsequent question box would appear asking what type of medication error was identified. The survey would continue in the same manner for remaining medications selected in alphabetical order. If the patient was over 65 years of age, the survey automatically included a third section for additional medications considered high-risk for the elderly patient population. The final portion of the survey inquired about the number of non-HR medications. Any medication not found in the HR medication list was assumed to be non-HR. Non-HR medications were not further classified into specific medications or drug classes, but the total number of medications and medication errors for non-HR medications were accounted for to estimate overall medications and medication errors. The survey displayed an auto-calculated total of HR and non-HR medications and errors to serve as a final-check by the technician before submitting the patient record via the survey tool.

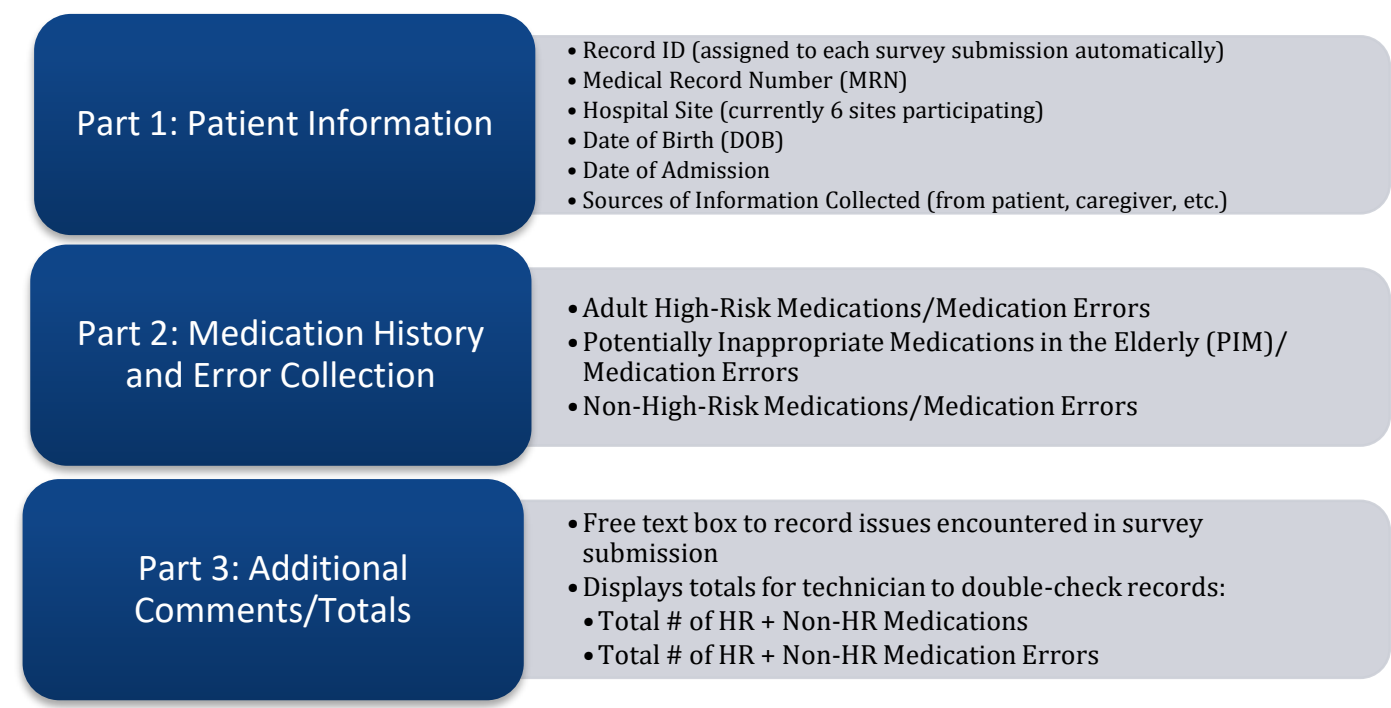

Figure 1. Ascension Texas Medication History Survey tool breakdown. Abbreviations used: HR, high-risk. 
Upon completion of a survey, each submission was automatically assigned a record number to code patient data and subsequently stored in the REDCap database. The pharmacist was able to view, edit, and add patient records. REDCap functionality also allowed custom report building with de-identified patient data, which was used to report information at a site-level as well as on a network level, accounting for all 6 participating hospital sites. Each report provided statistics on the total number and type of medications and medication errors, polypharmacy risk, and fall risk. These numbers were further broken down to specify the classes of HR medications, PIM medications, combined HR and PIM medications, non-HR medications, and overall medications and errors. Polypharmacy identified patients with $\geq 4$ high-risk medications and were therefore considered to have additional risk for medication-related adverse events. ${ }^{5-7}$ Fall risk identified those patients greater than 65 years of age that had $\geq 2$ CNSaffecting medications and were considered to have additional risk for falls and/or fractures as stated by the American Geriatrics Society 2019 Beers Criteria. ${ }^{26,40}$
A written training guide on completing the web-based survey tool was developed for the medication history technicians. Two medication history technicians were initially trained by a pharmacist on the web-based survey tool, during which a clinical pharmacist verified the accuracy of data documentation using the survey tool. Afterwards a clinical pharmacist trained the remaining four technicians on the survey tool, and the pilot program was incorporated into the call center workflow in April of 2019 to assess compatibility and outcomes.

\section{Results}

During April 2019 to October 2020, medication history technicians completed 191 medication history records using the Ascension Texas Medication History Survey tool, without the use of prior methods of recording data on paper. Out of a total of 1088 medications recorded, 448 medications were considered high-risk (41\%). The most common drug categories of high-risk medications included cardiovascular (39\%) and hypoglycemic drugs (13\%) (Table 1). From a total of 624 potential medication errors recorded, 261 were classified as potential high-risk medications errors (42\%) (Table 2).

Table 1. Classifying Potential High-Risk Medications and Medication Errors by Drug Category

\begin{tabular}{|c|c|c|c|c|}
\hline Drug Category & $n$ (Medications) & $\begin{array}{l}\% \text { (Total } \\
\text { medications) }\end{array}$ & $n$ (Errors) & $\%$ (Total errors) \\
\hline Cardiovascular & 175 & 39 & 101 & 39 \\
\hline Hypoglycemic & 58 & 13 & 40 & 15 \\
\hline CNS medications & 44 & 10 & 24 & 9 \\
\hline Antiplatelet (aspirin) & 39 & 9 & 23 & 9 \\
\hline Anticoagulant & 17 & 4 & 12 & 5 \\
\hline NSAIDs & 13 & 3 & 5 & 2 \\
\hline Corticosteroid (systemic) & 7 & 2 & 4 & 2 \\
\hline Antibiotic & 12 & 3 & 6 & 2 \\
\hline NTI & 7 & 2 & 5 & 2 \\
\hline Antiarrhythmic & 3 & 1 & 3 & 1 \\
\hline Cytostatic & 3 & 1 & 2 & 1 \\
\hline Immunosuppressant & 4 & 1 & 3 & 1 \\
\hline Antiretroviral & 6 & 1 & 3 & 1 \\
\hline Anti-rheumatic & 2 & 0 & 1 & 0 \\
\hline PIM CNS & 22 & 5 & 10 & 4 \\
\hline PIM Fall/Fracture & 15 & 3 & 9 & 3 \\
\hline PIM PPI & 12 & 3 & 6 & 2 \\
\hline PIM Anticholinergic & 7 & 2 & 3 & 1 \\
\hline PIM Endocrine & 2 & 0 & 0 & 0 \\
\hline PIM Antiplatelet & 0 & 0 & 0 & 0 \\
\hline PIM Electrolyte & 0 & 0 & 0 & 0 \\
\hline PIM Other & 0 & 0 & 0 & 0 \\
\hline Total HR Medications & 448 & 100 & 261 & 100 \\
\hline
\end{tabular}

Abbreviations used: NSAIDs, nonsteroidal anti-inflammatory drugs; CNS, central nervous system; NTI, narrow therapeutic index drugs; PIM, potentially inappropriate medication; PPI, proton-pump inhibitor

${ }^{a}$ CNS: Medications that affect the central nervous system without fall risk. Includes opioids, benzodiazepines, and first/second generation antipsychotics

bHypoglycemic: Insulin and sulfonylureas

'NTI: Narrow therapeutic index medications

dEndocrine: Includes thyroid, estrogen, progesterone, and testosterone hormone medications

ePIM CNS: Includes Adult CNS medications + antiepileptics, antidepressants, z-hypnotics, skeletal muscle relaxants, and meprobamate fPIM Fall/Fracture: Medications that affect the central nervous system and are associated with increased fall risk. Includes vasodilators, heral alpha blockers, and dipyridamole

gPIM Electrolyte: Includes desmopressin and stimulant laxatives

hPIM Other: Includes metoclopramide, mineral oil, and megestrol 
Figure 2: High-Risk Medication Errors Categorized By Error Type

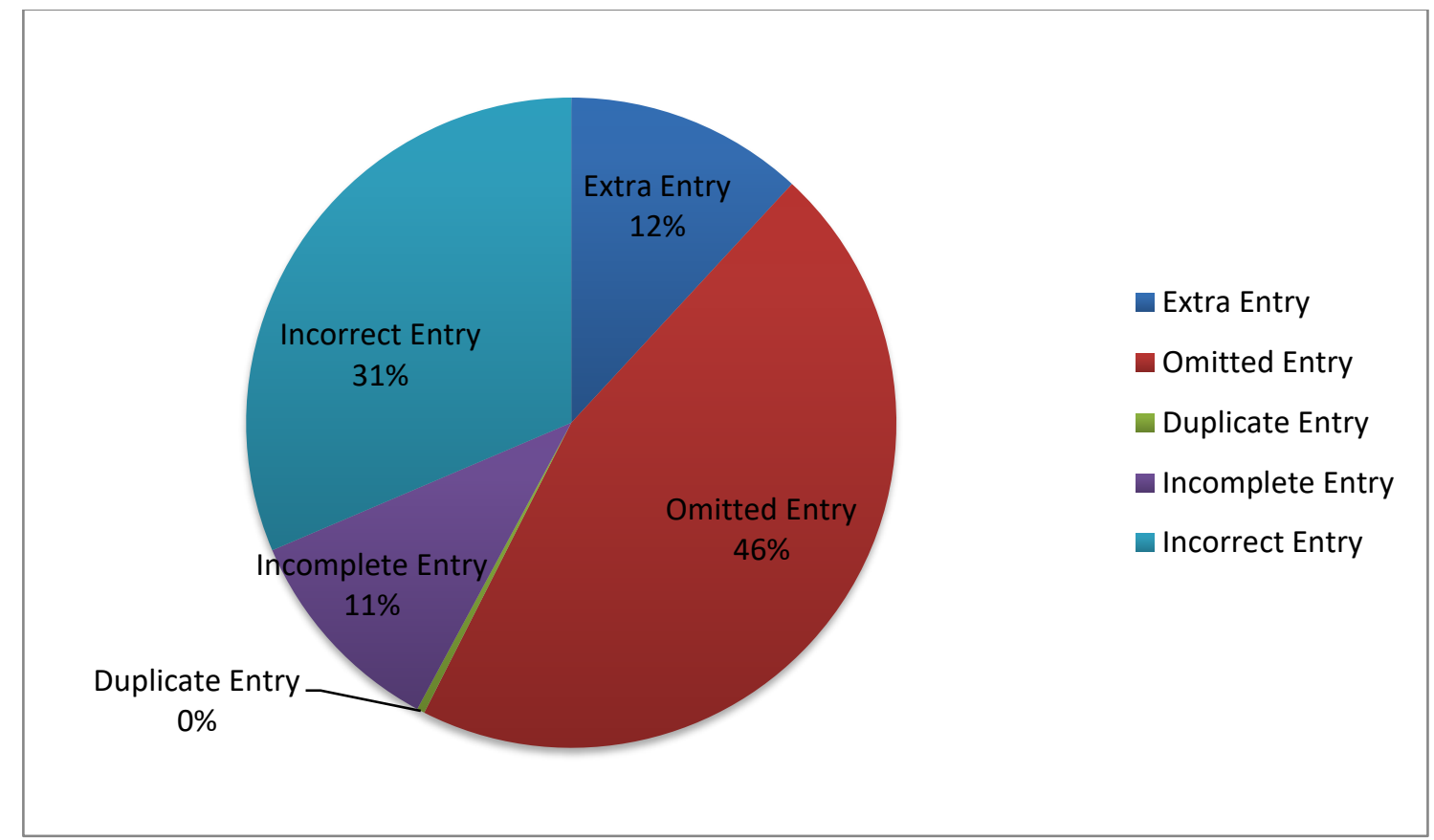

A total of 261 potential medication errors were identified from 191 patients in emergency departments of Ascension Texas from April 2019 to October 2020.

Table 2. High-Risk Medication Errors Categorized By Error Type

\begin{tabular}{|c|c|}
\hline Error type & $n$ \\
\hline Omitted Entry & 119 \\
\hline Incorrect Entry & 82 \\
\hline Extra Entry & 31 \\
\hline Incomplete Entry & 28 \\
\hline Duplicate Entry & 1 \\
\hline Other Entry ${ }^{a}$ & 0 \\
\hline Total & 261 \\
\hline
\end{tabular}

Data collected from 191 patients in emergency departments of Ascension Texas from April 2019 to October 2020. aOther: Error due to a medication allergy or intolerance listed in the patient's electronic medical record.

Data demonstrated that $58 \%$ of 175 cardiovascular-related prescriptions and $69 \%$ of 58 diabetes-related prescriptions were associated with a medication error. Additionally, 59\% of 39 aspirin prescriptions were associated with a medication error (Table 1). The most common types of medication errors were omitted entries (46\%), incorrect entries (31\%), and extra entries (12\%) (Figure 2), which were comparable to categories of medication errors reported in similar studies. ${ }^{4,5}$ The technicians were offered an option to pick "other error" in the survey tool, for exceptions to general error categories including a patient having an allergy to a certain drug that was on the medication list; however no "other errors" were identified (Table 2). Other variables in the survey tool identified that $27 \%$ of patients were considered to have polypharmacy and 2 patients were considered to have additional risk for falls and fractures. Potentially inappropriate medications in the elderly were found in 36 out of 80 patients (45\%), totaling up to 58 prescribed medications.

Results from this survey tool pilot period show that $58 \%$ of highrisk medications could potentially result in a medication error, and $57 \%$ of medication orders in general may potentially result in a medication error (Table 3). However, technicians are currently instructed to use the survey tool only for patients with potential medication errors found during medication history collection and therefore data does not reflect patients that had no identified potential medication errors. 
Table 3. High-Risk Medications and Medication Error Frequencies

\begin{tabular}{|l|l|}
\hline Total \# of HR Medications & 448 \\
\hline Total \# of All Medications & 1088 \\
\hline$\%$ of HR Medications & $41 \%$ \\
\hline & \\
\hline Total \# of HR Medication Errors & 261 \\
\hline Total \# of All Medication Errors & 624 \\
\hline$\%$ of HR Medication Errors & $42 \%$ \\
\hline & \\
\hline$\%$ HR medication orders that contain potential medication error & $58 \%$ \\
\hline$\%$ All medication orders that contain potential medication error & $57 \%$ \\
\hline
\end{tabular}

\section{Discussion}

The impact of accurate medication history and transitions of care is applicable to both the provider's accuracy in prescribing correct medication orders as well as how patients will take medications upon discharge. Arguably the most important benefit from reducing medication errors is decreasing preventable medication-related ADEs, hospital-stays, and improving patient quality of life. ${ }^{1-5}$ Data from a multistate patient safety organization database confirms that medicationrelated events are still a primary cause of patient safety incidents in the ambulatory care setting, from which $45.3 \%$ of incidents were accounted to medication-related errors. ${ }^{46}$ However, the cost of time and materials required to address a medication related-adverse event in hospital and outpatient systems is also substantial. Even for errors that do not result in harm or injury to the patient, studies show that each error requires an extra 20 minutes of time for healthcare professionals, of which most are pharmacists and nurses. ${ }^{3}$ Additional burdens related directly to the patient include lost earnings, inability to perform household duties, and compensation for pain and suffering. ${ }^{1,3}$

On average, 11 patient records were collected per month during the pilot period. Once the tool is integrated completely into the call center workflow, it is expected that at least 30 records will be completed per month, as evidenced from data collected prior to implementation of this survey tool. While a previous set of data collected manually over a period of 4 months obtained 124 records, the information was analyzed by a full-time pharmacist and pharmacy resident over the course of an entire year (April 2017 to August 2018), with severalmonth delays between initial data collection and final analysis. ${ }^{5}$ In comparison, this survey tool is built to auto-generate reports with numerous statistics, therefore time spent for analysis is negligent and can be gathered for any time frame of choice. Upon further training and workflow development, this tool could further be expanded to include all patients with a completed medication history in order to identify trends in high-risk medications, polypharmacy, and potential fall-risk in certain patient populations on a continuous basis.

The addition of this web-based survey tool allows for increased documentation of the interventions and impact offered by the telepharmacy services in Ascension Texas. Information collected is used not only to provide a more accurate medication record, but can lower the amount of medication discrepancies that often occur during patient discharge and follow through as the patient experiences transitions of care (TOC). 4,5,47 Accurate TOCs are a significant player in determining hospital readmission rates and can contribute to up to $20 \%$ of 30 -day readmissions and $34 \%$ of 90 -day readmissions. ${ }^{47}$ Based on published reports in 2006, annual gross savings to a hospital is estimated to be $\$ 3511$ per harmful error prevented. ${ }^{1}$ Inflating that cost to 2020 dollars estimates the cost-savings per medication error to be $\$ 4532 .{ }^{48}$ Therefore the economic impact of preventing 261 potential medication errors identified by medication history technicians using this survey tool equated to approximately $\$ 1,182,852$ (Table 4) in estimated savings for Ascension Texas in a span of 18 months. This value is estimated by capturing only a fraction of the patients for whom the call center completes medication histories and identifies potential errors for. Integration of the survey tool into regular workflow will likely identify nearly triple the amount of potential medication errors that are corrected before a patient is admitted into the hospital, totaling up to $\$ 2,365,704$ in potential cost avoidance in Ascension Texas per year (Table 4). Nationwide, identifying and resolving preventable medication errors in an outpatient setting is estimated to save an average of $\$ 3.5$ billion per year. ${ }^{1,3,8}$ 
Table 4. Economic Impact of Preventing Potential Medication Errors During Pilot Period

\begin{tabular}{|l|l|}
\hline Total \# of potential HR medication errors & 261 \\
\hline Average cost-savings per error (in-hospital) in 2020 & $\$ 4532$ \\
\hline Potential total cost savings to Ascension Texas in 18 months & $\$ 1,182,852$ \\
\hline Estimated total cost savings to Ascension Texas per year (post-pilot) & $\$ 2,365,704$ \\
\hline
\end{tabular}

The development of this survey tool posed a few limitations that may be useful to take into consideration. For the purposes of this study, non-HR medication information collected (ex. vitamins or supplements) was limited to total number of medications and medication errors identified, rather than type of medication. The purpose was to capture the total number of medications per patient, with a focus on high-risk medications due to their likelihood in causing significant harm, prolonged hospitalizations, or even death if used in error. However it should be recognized that non-HR medications used inappropriately can also cause medication errors and PADEs. Secondly, the survey tool does not identify high-risk medications for certain comorbid conditions, nor does it identify drug-drug interactions. These therapeutic concerns are to be evaluated by a physician or clinical pharmacist once a patient is admitted by using the reconciled medication list compiled by the medication history technician. Third, the classifications of HR medications in this survey tool are not automatically updated, and therefore must be manually adjusted as new medications or literature are approved. Future improvements may include a medication database that updates automatically with new FDA-approved medications, and further classifies medications into the appropriate drug class and highrisk designation. Lastly, the survey tool did not collect data on patients who had no potential medication errors found; therefore it was not possible to compare percentages of patients with potential errors vs. those without.

It is important to note that the incorporation of the pilot program into the call center workflow was hindered by the COVID-19 pandemic, and therefore the number of records collected over the set period of time was less than initially expected. However once established, the use of this virtual call center workflow and electronic collection of potential error identification can greatly impact healthcare processes that are trying to adapt to safer and web-based pharmacy practices to protect both patients and staff exposure to COVID-19 or similar conditions. This model can be successfully incorporated into a permanent workflow that can be prepared to function both inperson or virtually to access patients in hospital systems nationwide.

\section{Conclusion}

The Ascension Texas Medication History Survey tool is to be integrated completely into technician workflow by 2021. Data from the survey tool will additionally be used to generate monthly reports on a network level for pharmacy leadership that include numbers for HR and total \# of medications, as well as the top 2 categories of HR medications involved in medication errors. These results can identify areas of improvement for healthcare providers such as taking precaution in prescribing certain high-risk medications and further highlighting the importance of patient education and medication therapy management. Improved quality and efficiency of potential error identification will also aid clinical pharmacists and providers during the medication reconciliation process and result in fewer errors carried over during transitions of care.

Overall, the Ascension Texas Medication History Survey tool has significantly decreased the time and labor required to collect and report potential medication errors found during medication history collection. Potential adverse effects that might be avoided as a result of this error identification can greatly impact the cost of resolving medication-related adverse events in the Ascension Texas network of hospitals and prevent medication-related issues post-discharge. The adaptation of similar electronic data recording tools can be used in healthcare systems worldwide to decrease the healthcare costs associated with preventable medication-related ADEs and subsequent effects on patient morbidity, mortality and quality of life.

Acknowledgements: The authors thank Jordan Suh, Megan Campbell, An Nguyen, Kimberly Hodge, Lorena Sustaita, Tiffany Yoder, Araceli Dille, Jameela Cameron, and Laura Trujillo for their dedication and support of this work.

Funding/Support: None

Conflicts of Interest: None

\section{References}

1. Slight SP, Seger DL, Franz C, Wong A, Bates DW. The national cost of adverse drug events resulting from inappropriate medication-related alert overrides in the United States. J. Am. Med. Inform. Assoc. 2018 Sep;25(9):1183-8.

2. Hug BL, Keohane C, Seger DL, Yoon C, Bates DW. The costs of adverse drug events in community hospitals. Jt Comm J Qual Saf. 2012 Mar 1;38(3):120-6.

3. Aspden, Philip, and Philip Aspden. Preventing medication errors. National Acad. Press, 2007. 
4. Kramer JS, Stewart MR, Fogg SM, et al. A quantitative evaluation of medication histories and reconciliation by discipline. Hosp Pharm. 2014;49(9):826-838.

5. McGinnis, B., Padilla, E., Garret, P., \& Aziz, S. Using pharmacy technicians and telepharmacy to obtain medication histories in the emergency department. $J$ Am Pharm Ass. 2019; 59(3), 390-397.

doi:10.1016/j.japh.2019.01.019

6. Beuscart JB, Petit S, Gautier S, Wierre P, Balcaen T, Lefebvre JM, Kambia N, Bertoux E, Mascaut D, Barthélémy C, Cuny D. Polypharmacy in older patients: identifying the need for support by a community pharmacist. BMC geriatr. 2019 Dec 1;19(1):277.

7. Morrison et al. Promoting Safer Use of High-Risk Pharmacotherapy: Impact of Pharmacist-Led Targeted Medication Reviews. Drugs Real World Outcomes. 2015 Sep;2(3):2241-281.

8. Burton MM, Hope C, Murray MD, Hui S, Overhage JM. The cost of adverse drug events in ambulatory care. AMIA Symposium. 2007 Oct; p.90-93.

9. Pippins et al. Classifying and Predicting Errors of Inpatient Medication Reconciliation. J Gen Intern Med. 2008 Sep;23(9):1414-22

10. Mekonnen AB, McLachlan AJ, Brien JA. Pharmacy-led medication reconciliation programmes at hospital transitions: a systematic review and meta-analysis. J Clin Pharm Ther. 2016 Apr;41(2):128-44.

11. American Society of Health-System Pharmacists. ASHP statement on the pharmacist's role in medication reconciliation. Am J Health Syst Pharm. 2013;70(5):453-456.

12. Sachita S, Siemianowski L, Murphy M. Implementation of a pharmacy technician-centered medication reconciliation program at an urban teaching medical center. Am J Health Syst Pharm. 2014 Jan 1;71(1):51-56.

13. Balon J, Thomas SA. Comparison of hospital admission medication lists with primary care physician and outpatient pharmacy lists. J Nurs Scholarsh. 2011;43(3):292-300.

14. Pavlov A, Muravyev R, Amoateng-Adjepong Y, Manthous CA. Inappropriate discharge on bronchodilators and acid-blocking medications after ICU admission: importance of medication reconciliation. Respir Care. 2014;59(10):1524-1529.

15. Bond CA, Raehl CL. Clinical pharmacy services, pharmacy staffing, and hospital mortality rates. Pharmacotherapy. 2007;27(4):481-493.

16. PubMed. NIH National Center for Biotechnology Information, n.d. Available from: https://pubmed.ncbi.nlm.nih.gov/.

17. Saedder et al. Identifying high-risk medication: a systematic literature review. Eur J Clin Pharmacol. 2014 Jun;70(6):637-45.
18. Otero et al. Developing a list of high-alert medications for patients with chronic diseases. Eur J Intern Med. 2014 Dec;25(10):900-8.

19. Buajordet et al. Fatal adverse drug events: the paradox of drug treatment. J Intern Med. 2001 Oct;250(4):327-41.

20. Howard et al. Investigation into the reasons for preventable drug related admissions to a medical admissions unit: observational study. Qual Saf Health Care. 2003 Aug;12(4):280-5.

21. Lin et al. Potentially high-risk medication categories and unplanned hospitalizations: a case-time-control study. Sci Rep. 2017 Jan 23;7(1):1-9.

22. Blix et al. Drugs with narrow therapeutic index as indicators in the risk management of hospitalised patients. Phar Prac. 2010 JanMar;8(1):50-55

23. John et al. Antiplatelet agents do not impact the hospital course in patients with gastrointestinal bleeding. J Clin Gastroenterol. 2011 Aug;45(7):583589.

24. ISMP List of High-alert medications in acute care settings. Institute for Safe Medicine Practices (ISMP). 2018. Available from: www.ismp.org/MERP.

25. Budnitz et al. Emergency hospitalizations for adverse drug events in older Americans. N Engl J Med. 2011 Nov 24;365(21):2002-12.

26. Masud et al. Central nervous system medications and falls risk in men aged 60-75 years: the Study on Male Osteoporosis and Aging (SOMA). Age Ageing. 2013 Jan;42(1):121-4.

27. A to $Z$ List of Cancer Drugs [Internet]. National Cancer Institute. Available

from: https://www.cancer.gov/aboutcancer/treatment/drugs.

28. Stein, Stephanie Aleskow, Elizabeth Mary Lamos, and Stephen N Davis. A Review of the Efficacy and Safety of Oral Antidiabetic Drugs. Expert Opin Drug Saf. 2013; 12.2:153-175.

29. Ustulin, M., Woo, J., Woo, J. T., \& Rhee, S. Y. Characteristics of frequent emergency department users with type 2 diabetes mellitus in Korea. $J$ Diabetes Investig. 2018;9(2), 430-437.

30. Detournay, Bruno et al. Hypoglycemia Hospitalization Frequency in Patients with Type 2 Diabetes Mellitus: A Comparison of Dipeptidyl Peptidase 4 Inhibitors and Insulin Secretagogues Using the French Health Insurance Database. Vasc Health and Risk Manag. 2015; 11:417-425.

31. Anelli et al. Old and new antirheumatic drugs and the risk of hepatotoxicity. Ther Drug Monit. 2012 Dec;34(6):622-8.

32. Murdaca et al. Infection risk associated with anti-TNF$\alpha$ agents: a review. Expert Opin Drug Saf. 2015 Apr;14(4):571-82. 
33. CDC. Antibiotic Use in the United States, 2017: Progress and Opportunities. Atlanta, GA: US Department of Health and Human Services, CDC. 2017.

34. Shehab N, Lovegrove M, Geller A. US Emergency Department Visits for Outpatient Adverse Drug Events, 2013-2014. JAMA. 2016Nov22;316(20): 211525.

35. Zucker J, Mittal J, Jen SP, Cheng L, Cennimo D. Impact of Stewardship Interventions on Antiretroviral Medication Errors in an Urban Medical Center: A 3Year, Multiphase Study. Pharmacotherapy. 2016 Mar 6; 36(3):245-51.

36. USP Therapeutic Categories Model Guidelines. FDA. 2018 Mar 28. Available from: https://www.fda.gov/regulatory-information/fdaaaimplementation-chart/usp-therapeutic-categoriesmodel-guidelines.

37. AHFS Classification - Drug Assignments. AHFS Clinical Drug Information. 2020 Feb 6. Available from: https://www.ahfsdruginformation.com/ahfsclassification-drug-assignments/.

38. Clark CM, Shaver AL, Aurelio LA, Feuerstein S, Wahler Jr RG, Daly CJ, Jacobs DM. Potentially Inappropriate Medications Are Associated with Increased Healthcare Utilization and Costs. J Am Geriatr Soc. 2020 Aug 5.

39. Weir DL, Lee TC, McDonald EG, Motulsky A, Abrahamowicz M, Morgan S, Buckeridge D, Tamblyn R. Both new and chronic potentially inappropriate medications continued at hospital discharge are associated with increased risk of adverse events. J Am Geriatr Soc. 2020 Mar 31.

40. American Geriatrics Society 2019 Update Expert Panel. American Geriatrics Society 2019 Updated Beers Criteria for Potentially Inappropriate Medication Use in Older Adults. J Am Geriatr Soc. 2019Jan01;00(00):00-21. DOI: 10.1111/jgs.15767.
41. O'Mahony D. STOPP/START criteria for potentially inappropriate medications/potential prescribing omissions in older people: origin and progress. Expert Rev. Clin. Pharmacol. 2020 Jan 2;13(1):15-22.

42. Choosing Wisely. Ten Things Clinicians and Patients Should Question. American Geriatrics Society. 2015.

43. Kim et al. Development of a List of Potentially Inappropriate Drugs for the Korean Elderly Using the Delphi Method. Healthc Infor Res. 2010; 16(4): 231252.

44. PA Harris, R Taylor, R Thielke, J Payne, N Gonzalez, JG. Conde, Research electronic data capture (REDCap) A metadata-driven methodology and workflow process for providing translational research informatics support. J Biomed Inform. 2009 Apr;42(2):377-81.

45. PA Harris, R Taylor, BL Minor, V Elliott, M Fernandez, L O’Neal, L McLeod, G Delacqua, F Delacqua, J Kirby, SN Duda, REDCap Consortium, The REDCap consortium: Building an international community of software partners. J Biomed Inform. 2019 May 9 [doi: 10.1016/j.jbi.2019.103208]

46. Sharma AE, Yang J, Del Rosario JB, Hoskote $M$, Rivadeneira NA, Council SR, Sarkar U. What Safety Events Are Reported For Ambulatory Care? Analysis of Incident Reports from a Patient Safety Organization. Jt Comm J Qual Saf. 2020 Aug 21.

47. Jencks SF, Williams MV, Coleman EA. Rehospitalizations among patients in the Medicare fee-for-service program. N Engl J Med. 2009;360(14):1418-1428.

48. Inflation Calculator: Find US Dollar's Value from 19132020. US Inflation Calculator. 2020 Dec 10. Available from: https://www.usinflationcalculator.com/. 